\title{
Procedimiento para implementar la gestión del conocimiento en el comercio minorista de bienes
}

\author{
Raúl Yoel La Fé Jiménez* \\ Facultad de Ciencias Económicas \\ Universidad Central "Marta Abreu" de Las Villas
}

\section{Resumen}

La presente investigación tiene como objetivo diseñar un procedimiento para la gestión del conocimiento en la sucursal ARTex de Villa Clara (Cuba), empresa con una línea de negocio de comercio minorista de bienes, con el fin de incrementar su eficiencia.

Se realizó una exhaustiva revisión bibliográfica enfocada en la gestión del conocimiento y los conceptos que de ella se derivan; se aplicaron encuestas a una cantidad significativa de expertos, clientes especializados y trabajadores de la entidad y se utilizaron técnicas como la entrevista y la observación. El estudio es de gran utilidad para la sucursal ARTex de Villa Clara pues se enfoca en sus procesos clave: gestión de la oferta, diseño del punto de venta y atención al cliente.

\section{Palabras clave}

Procedimiento, gestión, conocimiento, comercio minorista.

Recibido: 17/11/2013 - Aceptado: 25/02/2014

*Correo electrónico: raulfj@uclv.edu.cu 


\title{
A procedure to implement Knowledge Management in the goods retail sector
}

\author{
Raúl Yoel La Fé Jiménez* \\ Facultad de Ciencias Económicas \\ Universidad Central "Marta Abreu" de Las Villas
}

\begin{abstract}
This research was conducted at the Villa Clara Branch of ARTex, a retail business, with the purpose of designing a knowledge management method to increase efficiency.

An exhaustive literature review focused on knowledge management and the concepts derived from this was performed; a significant quantity of surveys to experts, specialized customers and employees of the entity were applied, and techniques such as interviews and observations were used. The research is useful for ARTex Branch of Villa Clara as it focuses on key processes: supply management, point of sale design and customer service.
\end{abstract}

\section{KEY WORDS}

Procedure, management, knowledge, retail.

*E-mail: raulfj@uclv.edu.cu 


\section{Introducción}

En la actualidad, el rol del conocimiento en las organizaciones ha cambiado debido a los paradigmas asociados a la nueva economía basada en el conocimiento, por lo que se hace imprescindible aprender a manejar este activo a fin de satisfacer las necesidades del cambiante mercado. Se debe gestionar lo que saben las personas que integran la empresa y conseguir que la organización aprenda desarrollando el conocimiento de todos y generando nuevas formas de aprender a aprender. Por ello es necesario estudiar el conocimiento organizacional de la empresa.

La implementación de la gestión del conocimiento (cuyo objetivo básico es organizar y aplicar los recursos externos e internos de la organización para permitirle operar, aprender y adaptarse a los cambios del entorno) es la principal herramienta para averiguar cómo hacer las cosas de la manera más eficiente, generar ventajas competitivas a partir de los procesos de innovación y explotar las capacidades de cada miembro de la entidad.

Cuba posee una verdadera riqueza nacional en cuanto a conocimientos e instrucción, producto de un intensivo programa educativo desarrollado en las últimas décadas; sin embargo, existe insatisfacción con los resultados económicos y productivos logrados por las organizaciones, lo que evidencia el desaprovechamiento de esa riqueza.

En Cuba, las empresas estatales de comercio minorista de bienes tienen la misión de satisfacer las necesidades de bienes de los consumidores. Este sector es el mediador entre el sector industrial y el mercado. La comunicación entre ambos agentes económicos es tarea del comercio. Un sector ya existente, pero ahora renovado con los Lineamientos de la Política Económica y Social, ha crecido en los últimos dos años con los trabajadores por cuenta propia que han invadido las calles con un comercio agresivo y complaciente para el consumidor.

La sucursal ARTex de Villa Clara cuenta con un personal altamente capacitado en la venta de artesanías y bienes culturales. Es una organización con presencia en toda la provincia y con oportunidades de crecimiento por el incremento del turismo en su territorio. La situación problémica, o el conjunto de problemas que subsisten en el objeto de la investigación, en este caso radica en la falta de aprovechamiento del potencial de conocimientos en la sucursal ARTex de Villa Clara. A pesar de la formación del personal, los clientes se 
muestran insatisfechos con su servicio. Esta entidad del estado ha visto disminuidas sus ventas a pesar de tener más experiencia en el ramo y estar mejor posicionada que los cuentapropistas. No utiliza su potencial de conocimiento para enfrentar los cambios que ocurren por la dinámica del mercado, pues los procesos de generación y explotación del conocimiento no están organizados, y la cultura instaurada no ayuda a su uso efectivo, por lo que existen ventajas que no han sido aprovechadas.

El problema científico que se plantea es: ¿cómo contribuir, mediante el diseño de un procedimiento para la gestión del conocimiento, a la eficiencia de esta empresa?

De ahí que el objetivo general de la investigación sea diseñar un procedimiento para la gestión del conocimiento en la sucursal ARTex de Villa Clara. Por lo tanto, la hipótesis es: si se diseña un procedimiento para gestionar el conocimiento en la sucursal ARTex de Villa Clara será posible mejorar su eficiencia.

Para llevar a cabo la investigación se revisaron diversas fuentes bibliográficas y se recopiló información y se estableció una relación directa con sus empleados. Los resultados del procedimiento explican la urgencia de aprovechar el conocimiento al máximo en la empresa y diseñar nuevos espacios para la creación del mismo, en aras de hacerla más competitiva.

\section{La gestión del conocimiento en las organizaciones}

La gestión del conocimiento es una ciencia relativamente nueva que se ha desarrollado con más fuerza en los años noventa y a partir del 2000. El conocimiento siempre está en relación con la información. "Conocimiento e información son dos conceptos que están íntimamente relacionados, pues el conocimiento no trabaja en vacío sino que funciona siempre procesando información [...] el conocimiento capacita a las personas para actuar y dirigir inteligentemente a partir de las fuentes de información disponibles” (Benavides Velasco y Quintana García, 2003: 12). Esto es que no se admite una gestión del conocimiento en una empresa donde la gestión de información es deficiente o donde no se le da el valor que merece. En este sentido puede decirse que:

La infraestructura tecnológica debe facilitar los flujos de información y la comunicación interactiva entre las personas y grupos que integran la organización en 
todo momento. El problema consiste en saber cómo emplear las denominadas TICs para aprender más y mejor y cómo utilizarlas para realizar una gestión eficaz del conocimiento. Las tecnologías de la información dinamizan la transmisión de la información pero la gestión eficaz del conocimiento depende de la cognición humana y de la comunicación. Para lograrla, se necesita conocer y desarrollar una cultura de la información, así como ajustar los procesos de comunicación e información a las características específicas de las personas y grupos; a ello se le conoce genéricamente como personalización de la información [Núñez Paula y Núñez Govín, 2005: 3].

Ahora bien, el conocimiento es la base de toda actividad empresarial; la capacitación de las personas en los temas referentes al puesto de trabajo es vital en la conformación del conocimiento organizacional. "El conocimiento en las empresas es un conjunto abierto de ideas, creencias y experiencias contrastadas, y asimiladas mediante la reflexión que sirve de guía para la acción. Se encuentra incrustado en los individuos, los grupos, las organizaciones y el entorno social” (Riesco González, 2006: 66). En esta definición se incluye la diseminación del conocimiento en los diferentes niveles en los que puede encontrarse. Los individuos son el núcleo básico y la fuente principal del conocimiento, la coordinación entre ellos en función de objetivos organizacionales específicos estructura el conocimiento de los grupos. A su vez, el conocimiento de las organizaciones es la sistematización de los conocimientos de los individuos y de los grupos. Finalmente, el entorno es un receptor del conocimiento que se genera y que portan las organizaciones y es utilizado para el beneficio social. Sobre lo anterior, es posible afirmar que:

el conocimiento es reconocido como un producto social, por cuanto se construye socialmente como un producto de la actividad de los hombres, en donde cada individuo adquiere gran parte de su saber a partir de su propia experiencia, pero considerando una asociación con los demás y un aprendizaje inteligente desde esa cotidianidad. Este saber es acumulado por la sociedad y distribuido, en forma explícita e implícita [Ollarves Levison, Estévez González y Salguero, 2012: 20].

Según la cita anterior, el conocimiento tiene dos formas principales de manifestarse en el entorno y en las organizaciones: tácito y explícito. El conocimiento tácito se define como "el que poseen las personas, es inseparable de su experiencia y puede ser compartido e intercambiado, principalmente mediante 
contactos directos" (Ponjuán Dante, 2006: 13). Asimismo se ha dicho que es un conocimiento "altamente personal, muy difícil de formalizar y expresar, consistente en modelos mentales, creencias y perspectivas que dirigen la acción” (Pérez Fernández, 2007: 17). Este conocimiento, de alto valor para la conformación del conocimiento explícito, no es fácilmente transferible y por lo tanto no permanece en la organización cuando los portadores se marchan.

Por otro lado, el conocimiento explícito es "formal y sistemático, fácil de comunicar y compartir usando lenguaje científico, un plano o unas especificaciones de producto" (Pérez Fernández, 2002: 17). Además se sabe que "puede ser transferido de un individuo a otro usando algún sistema de símbolos [...] Es un conocimiento que ha sido codificado de alguna manera y puede comunicarse 0 difundirse con facilidad" (Ponjuán Dante, 2006: 13). Por su carácter transferible y permanente en la empresa es más valioso para esta.

De igual modo debe apuntarse que:

el conocimiento [...] se concibe como el activo más importante en las organizaciones, así como un producto y una fuente de ventajas competitivas, innovación, desarrollo e ingresos. Para elevar la capacidad creativa e innovadora del individuo en el colectivo, es necesario una gestión consciente y planificada del conocimiento, que potencie su creación, transferencia, conservación y reutilización en diferentes contextos [Núñez Paula y Núñez Govín, 2005].

Por lo que, desde el punto de vista económico, resulta ventajoso para las empresas dedicar esfuerzos a la gestión del conocimiento, con la inherente autorrealización que tal proceso conlleva en las personas.

Con base en lo anterior, la gestión del conocimiento "es la función que planifica, coordina y controla los flujos de conocimientos, estando formada por varios procesos interrelacionados tales como el proceso de adquisición de conocimiento, procesos de creación de conocimiento y proceso de internalización de conocimiento" (García Morales, 2005: 65). Así, hay funciones de gestión del conocimiento que se consideran elementales: adquisición, creación, almacenaje y comunicación. Además, se sabe que "es el proceso de crear valor a través del uso de activos intangibles de la empresa, es la transformación de la información en conocimiento y del conocimiento en negocio" (Rezende, 2001: 53).

Por otro lado, Orozco Silva y Carro Suárez proponen que la gestión del conocimiento "reconoce y utiliza el valor más importante de las organizaciones: 
el recurso humano y el conocimiento que los humanos poseen y aportan a la organización” (2002: 18). Así que esta ciencia da un valor fundamental a los recursos humanos y sus capacidades, y al hablar de la dirección o gerencia de la gestión del conocimiento se debe decir que "es la estrategia que utilizan las organizaciones para lograr que el conocimiento permanezca en la organización y no en personas, es decir, que el saber hacer de la organización no recaiga en cabeza de personas sino de procesos” (Alzate Molina y Gallego Álvarez, 2012).

\section{Procedimiento para la gestión del conocimiento en el comercio minorista de bienes en la sucursal ARTex de Villa Clara}

El procedimiento que se propone tiene como objetivo utilizar la gestión del conocimiento para mejorar la eficiencia y la eficacia de la sucursal ARTex de Villa Clara. Está basado en el modelo de gestión del conocimiento planteado por el autor para las empresas de comercio minorista de bienes.

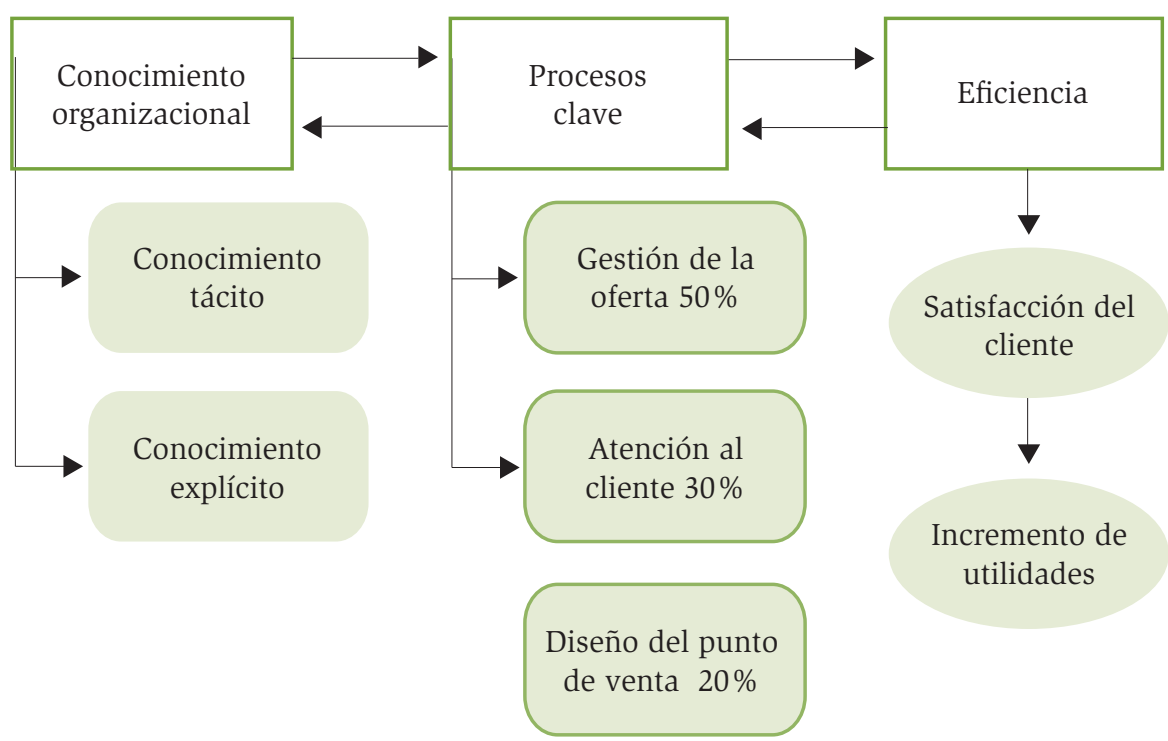

Fuente: La Fé Jiménez (2013).

Figura 1. Modelo de gestión del conocimiento para el comercio minorista 
Este modelo consta de una clasificación de los conocimientos tácitos y explícitos que influyen en los procesos clave del comercio minorista de bienes: gestión de la oferta, atención al cliente y diseño del punto de venta. A su vez, el resultado de esta influencia se refleja en dos indicadores generales de eficiencia: satisfacción del cliente e incremento en las utilidades.

Para aplicar el procedimiento en cualquier empresa de comercio minorista de bienes se deben tener presentes las premisas siguientes:

1. Las empresas de comercio minorista de bienes cuentan con un amplio conocimiento organizacional creado que no es utilizado al máximo de sus posibilidades.

2. Existen procesos internos que determinan la satisfacción del cliente y el logro de rendimientos económicos para las empresas, según el modelo citado.

3. La eficiencia de la empresa puede medirse por la satisfacción del cliente como una de las metas fundamentales.

4. La efectividad de la empresa puede medirse por los rendimientos económicos, considerando la diferencia entre los ingresos totales que genera la utilización del conocimiento y los costos de aplicar el procedimiento en general.

El procedimiento consta de cinco etapas y 12 fases con una secuencia lógica y retroalimentación constante. Las etapas 1 a 3 son la base de las 4 y 5. La etapa 2 es condición para la 3 y la etapa 5 se realiza simultáneamente con la 4 . La etapa 5 retroalimenta a todas las anteriores. Las etapas y fases se explican en detalle a continuación.

\section{Etapa 1. Diagnóstico de gestión del conocimiento}

FASE 1. Caracterización estratégica de la actividad empresarial. Se analiza la empresa en cuanto a la planificación estratégica: misión, visión, matriz DAFO, objetivos estratégicos y la proyección en ellos de finalidades que enfaticen el conocimiento o la limitación en ese aspecto. Esto se hace para alinear mejor la estrategia general de la empresa con la gestión del conocimiento.

FASE 2. Análisis del sistema de información. Debe analizarse la estructura del sistema general de información en la empresa objeto de estudio: si se utilizan 
plataformas como intranet o extranet en la distribución de la información y la efectividad de su empleo, los programas computacionales que usan para el procesamiento de la información y si los individuos que los administran y quienes los utilizan están sintonizados y en coherencia.

FASE 3. Determinación de las oportunidades de aprendizaje. Estas pueden existir de manera inconsciente y deben determinarse para tener mayor conciencia de sus posibilidades. Pueden ser espacios virtuales de comunicación, como el correo electrónico, foros, plataformas interactivas y no virtuales como cursos de formación y capacitación, reuniones periódicas, publicaciones y otras a las que el personal esté habituado y a las cuales solo habría que modificar en cuanto a su forma.

\section{Etapa 2. Determinación de los conocimientos críticos}

FASE 1. Determinación de las formas en que se manifiesta el conocimiento. Estas se deben tener en cuenta en general según se muestra en el cuadro 1.

\section{Cuadro 1. Clasificación del conocimiento}

\begin{tabular}{ll} 
Conocimiento TÁcito & ConocimiEnTO EXPLícito \\
\hline Habilidades y capacidades & Manuales \\
\hline Formación & Relaciones internas \\
Experticia & Relaciones con el entorno \\
\hline
\end{tabular}

Fuente: Elaboración propia.

Por cada área de la empresa deben determinarse las formas específicas en que se manifiesta el conocimiento. Esta fase debe ser lo más exhaustiva posible y quedar reflejada en un mapa de conocimiento.

FASE 2. Descripción de los conocimientos críticos. A partir de la influencia de cada conocimiento en los procesos clave de la empresa, deben seleccionarse los conocimientos más influyentes. Luego debe hacerse una descripción más profunda de los mismos en el orden de prioridad o importancia.

Esto debe combinarse con la influencia que tiene cada proceso sobre los resultados de la empresa, para saber el orden de prioridad de los procesos en la gestión del conocimiento. 


\section{Etapa 3. Evaluación del conocimiento}

Fase 1. Adecuación y aplicación de las técnicas de evaluación. Cada uno de los conocimientos críticos seleccionados en la etapa anterior deben evaluarse en cuanto a su nivel de aprovechamiento y su adecuación a las exigencias de la empresa.

FASE 2. Análisis del estado del conocimiento en la empresa. Por cada tipo de conocimiento tácito o explícito deben redactarse las debilidades y las fortalezas detectadas. En este caso, se entiende por debilidad las insuficiencias en la conformación o el aprovechamiento del conocimiento. Como fortaleza se entiende todo potencial de los conocimientos existentes en la empresa. Debe realizarse un análisis general de las debilidades y las fortalezas para definir la estrategia en la etapa siguiente.

\section{Etapa 4. Estrategia de gestión del conocimiento}

FASE 1. Diseño de la estrategia. A partir de las etapas anteriores debe elaborarse una estrategia general que permita aprovechar las potencialidades de la empresa en términos de gestión para el aprendizaje y la utilización consciente de los conocimientos, y para explotar las fortalezas con el fin de atenuar las debilidades. La estrategia debe seguir la lógica siguiente:

Cuadro 2. Matriz de convergencia estratégica conocimiento-proceso clave

\begin{tabular}{|c|c|c|c|}
\hline & GESTIÓN DE LA OFERTA & Atención AL CLIENTE & $\begin{array}{l}\text { DISEÑO DE LOS } \\
\text { PUNTOS DE VENTA }\end{array}$ \\
\hline CONOCIMIENTO TÁCITO & I & II & III \\
\hline CONOCIMIENTO EXPLÍ́ITO & VI & $\mathrm{V}$ & VI \\
\hline
\end{tabular}

Fuente: Elaboración propia.

Para cada cuadrante de convergencia del conocimiento con los procesos de éxito deben efectuarse estrategias que contribuyan a la eficiencia de la empresa a partir del uso consciente de los conocimientos. La estrategia necesita contener:

- Objetivos a cumplir en cuanto al conocimiento organizacional 
- Acciones que cumplan los objetivos y potencien los resultados de la empresa

La estrategia plasmada en cada cuadrante se realizará mediante las siguientes técnicas de gestión del conocimiento:

1. Sistemas de información

2. Oportunidades de aprendizaje

3. Comunidad de práctica

4. Coaching

5. Capacitación

6. Directorio de expertos

7. Lecciones aprendidas

Se toma como base el funcionamiento de los sistemas de información y los espacios de aprendizaje para crear comunidades de práctica que se nutran de los directorios de expertos como facilitadores del conocimiento y utilicen la capacitación o entrenamiento en el marco de la comunidad. El coaching y las lecciones aprendidas pueden ser el medio de supervisión sistemática y operativa de la estrategia.

Sistema de información y espacios de aprendizaje de la empresa

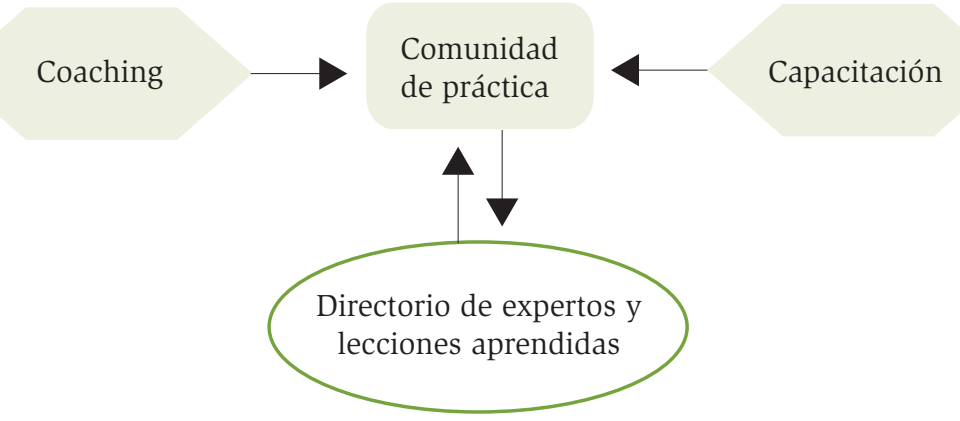

Fuente: Elaboración propia.

Figura 2. Propuesta de técnicas de gestión del conocimiento 
FASE 2. Aprobación y aplicación de la estrategia. Se debe consultar a los directivos de la empresa para que den su aprobación sobre la estrategia y hagan los reajustes requeridos para su aplicación, que debe organizarse en cada una de las técnicas con responsables y tiempos definidos, así como con los recursos necesarios para ello. En la medida de lo posible, deben introducirse a los procesos y a las estructuras habituales de la empresa, de acuerdo con los pasos siguientes:

1. Explicar las técnicas a los miembros de la empresa y justificar la implementación para que no resulte una imposición y pueda crearse el ambiente de aprendizaje propicio.

2. Organizar la aplicación: designar responsables y estimar los recursos que la empresa puede emplear para que la estrategia resulte en mayor eficiencia general.

3. Prueba piloto: ensayos de aplicación de técnicas con conocimientos más fáciles de comprender y aprovechar, después ir aumentando el rigor y la apropiación de otros más complejos y creando conocimiento más pertinente.

4. Implementación definitiva: aplicar todas las técnicas a la vez e incorporarlas definitivamente a las estructuras de la empresa.

\section{Etapa 5. Evaluación del conocimiento organizacional}

FASE 1. Determinación de la eficiencia por el cliente. Debe hacerse antes de poner en práctica la estrategia, en el orden siguiente:

1. Definir la muestra de clientes especializados a emplear: deben ser mayores de edad, clientes habituales de la empresa y con formación en temas de comercialización o con un amplio dominio de la oferta y los puntos de venta de la empresa para que puedan dar criterios que sirvan como base para los reajustes. Deben comprometerse a participar en el experimento y responder de forma crítica antes y después de la aplicación de la estrategia. La muestra debe ser representativa y los clientes no deben pertenecer a otras empresas del sector ni a la que se está investigando para que no haya predisposiciones. 
2. Determinar el coeficiente de satisfacción del cliente previo a la implementación: elaborar los cuestionarios y precisar sus incongruencias mediante muestras piloto. Se aplicará el test a partir de la idea global de satisfacción con los servicios en la empresa antes de llevar a cabo la estrategia. Tabular los resultados por cada criterio y cliente. El coeficiente se calcula sumando todos los valores de la matriz que se forma y dividiendo entre la cantidad de casillas. Debe hacerse por cada proceso clave y en general. Los pesos específicos que se han asignado responden al valor dado a cada proceso en la satisfacción del cliente.

3. Determinar el coeficiente de satisfacción después de la implementación: debe realizarse por proceso clave y en general para hacer comparaciones por cada uno, y tienen que usarse los mismos clientes que al principio. Se calcula de la misma manera que la implementación y se compara para ver el cambio positivo. Los valores deben estar entre 1 y 5.

FASE 2. Análisis costo-beneficio. Medirá el impacto económico de la gestión del conocimiento y debe comenzar desde la etapa 1 de acuerdo con los pasos que se enumeran a continuación:

1. Determinar los costos de implementación por etapas y fases en partidas adecuadas: calcular todos los gastos de materiales, horas de trabajo de empleados y otros en que pueda incurrirse por la aplicación de todas las etapas anteriores con sus fases.

2. Estimar los ingresos por utilización del conocimiento: calcular el incremento de los ingresos por aplicar las técnicas de aprovechamiento del conocimiento en la empresa; debe hacerse con la ayuda de los especialistas de economía y de comercialización en conjunto.

3. Calcular la ganancia o pérdida: calcular el resultado obtenido como valor agregado por la aplicación de las técnicas de aprovechamiento del conocimiento.

FASE 3. Retroalimentación de las etapas anteriores. Los resultados de la evaluación deben contribuir a identificar nuevos conocimientos que se plantaron en la organización y a determinar el impacto de la gestión del conocimiento en la misma. Puede haber nuevos espacios de aprendizaje y nuevas tareas por realizar. 


\section{Aplicación del procedimiento en la sucursal ARTex de Villa Clara}

La sucursal ARTex de Villa Clara se funda el 26 de abril de 1992 como una prueba de mercado que auguró magníficos niveles de ingresos. En correspondencia con el grado de desarrollo de la empresa y con las condiciones de los territorios se han ido fundando sucursales en distintas provincias: en la región central surgieron primero las de Sancti Spíritus y Cienfuegos; se comenzaron a vender discos y casetes en la Casa de las Grabaciones, ubicada en el parque Las Arcadas de Santa Clara; estos y otros productos vinculados a la figura del Che se vendían en el Museo de la Plaza. Con un mayor surtido se abrió un punto de venta en el cine Camilo Cienfuegos.

La sucursal ARTex de Villa Clara posee una estructura organizacional horizontal, integrada en el primer nivel por el gerente general y en el segundo por las gerencias de Recursos Humanos, Comercial, Económica, Comunicación y Marketing, la Administración y la Gerencia de Centros Culturales. Trabajan por consolidar cada día los vínculos con el sistema de cultura en el territorio. Todas las instituciones de la cultura que se relacionan con ella mantienen estrechos vínculos a través de sus funciones metodológica, de capacitación o comercial, según corresponda.

\section{Diagnóstico de gestión del conocimiento}

Algunos aspectos evidencian que en la organización se tiene en cuenta el conocimiento, pero se aplica de forma desorganizada, es decir, sin un proceso de gestión del mismo. La entidad ha creado burós de turismo en dos localidades con el fin de brindar información de sus ofertas a quien lo requiera; todas sus acciones tienen como función central la comercialización de sus productos y su arma fundamental es el reconocimiento y la entrega de información al público, para esto llevan a cabo ferias, eventos, exposiciones y modelajes. Ponen un cúmulo de equipos con el fin de promocionarse; fomentan el conocimiento a la hora de plasmar pinturas cubanas en sus artesanías (muchas de ellas ya conocidas y otras que salen a la luz por primera vez); guían sus esfuerzos hacia la edición y comercialización de libros, folletos, revistas y vivencias; e imparten conferencias, fundamentalmente a extranjeros, contando lo más importante de nuestra cultura y promocionando imágenes. ARTex es una gran estrategia de divulgación de las raíces cubanas. 
Sus proyecciones elaboradas para el quinquenio ponen de manifiesto su visión futurista, al darle un papel primordial a la consolidación de la comunicación, permitiéndole apuntalar su imagen y sus acciones comerciales. Además, se proponen continuar trabajando para lograr que la informática y las comunicaciones se conviertan en una herramienta de trabajo y funcionamiento en la empresa, interviniendo en los procesos de control, gestión, administración y dirección, y propiciando que la información sea coherente, fidedigna y oportuna, así como una herramienta en la toma de decisiones empresariales.

Fuera de la empresa, es decir, en el sistema de tiendas, utilizan el acceso al servidor ftp de la casa matriz, en La Habana, mediante un módem que les permite acceder a la plataforma de acceso público. También echan mano del correo electrónico y del teléfono y para el procesamiento de los datos recurren a los programas de venta por caja (o venta automatizada) y la ofimática.

Esta sucursal usa el sistema integral Zeus, conformado por una serie de programas empleados por los departamentos de economía, recursos humanos, comercial y centros culturales. Todos los programas de este sistema son creados por ARTex y son: sistema de inventario general, caja y banco, cobros y pagos, sistema de planes, de medios básicos, de útiles, nómina, personal prenómina, nómina de estimulación, comercial y codifica. Los principales problemas encontrados en ellos son:

- No son tecnología cliente-servidor; se trabaja directamente sobre el servidor, lo cual produce que se sobrecargue y dificulte el funcionamiento de la red de la entidad.

- La base de datos no es única, cada sistema tiene sus tablas independientes, lo que provoca constantes exportaciones e importaciones de información, saturando el servidor.

- No están certificados.

- Debido al nivel de resumen de los resultados, no permiten su interpretación inmediata por los especialistas, economistas y contadores.

El flujo de información de la entidad es muy abarcador: tiene como elementos rectores la contabilidad, ya que todo contribuye a esta, desde los contratos a los artesanos y las nóminas hasta la realización de los planes empresariales y la codificación, pues es la vía por la cual la casa matriz controla los inventarios y la diferenciación de los productos. 
Una de las oportunidades de aprendizaje que posibilita la comunicación, el intercambio de conocimientos, son las reuniones en la entidad; el Consejo de Administración realiza una reunión ordinaria mensual y por lo común se reúne en sesiones extraordinarias semanales. Las áreas se juntan una vez al mes de forma programada y pero también se reúnen siempre que es necesario. Todos los gerentes de área y los participantes en el Consejo de Administración despachan cada mes con el gerente general y se lleva a cabo una lluvia de ideas en aras de resolver los problemas encontrados. Las soluciones a estos pueden o no salir de estas reuniones.

Los cursos de habilitación que se han impartido son: curso de dinero encaminado sobre todo a los encargados de la comercialización de los productos, que enseña habilidades como la identificación de dinero falso y reconocimiento de firmas, y brinda herramientas útiles para su fácil manipulación; el curso de inspectores generales, como bien dice su nombre, proporciona los conocimientos exactos para lograr un efectivo proceso de control; existen asimismo los cursos de formación de inspectores sindicales sociales, el curso para dependencia de ARTex, el curso básico de sonido y el curso nacional de centros culturales y de vendedores, este último dura alrededor de cuatro meses y, al igual que todos, luego de un tiempo se revisa su impacto en el proceso de evaluación del desempeño.

Por otra parte, a los trabajadores de reciente ingreso a la cadena de tiendas de ARTex se les imparte un pequeño curso de cuatro horas en el que se les presentan todos los directivos, ya sea personalmente o por fotos; se les explica la estrategia general de la empresa, se les da a conocer los valores, la historia, sus futuras obligaciones, las bondades que brinda la entidad y los sistemas que emplea.

ARTex trabaja en sus puntos de venta con la premisa de que sus vendedores sean integrales, es decir, que todos conozcan por igual las características de los productos que venden. Para esto organiza cursos intensivos de habilitación, con el fin de ampliar el horizonte cultural de sus vendedores, pretende convertirlos en promotores culturales que desarrollen habilidades para vender y para incentivar al cliente; su objetivo es que el cliente no solo se lleve a casa un bien material sino que sea portador de un cúmulo de conocimientos que tengan como eje la cultura cubana. Entre los cursos que programan se encuentran: 
- Curso técnico de reciclaje. Permite conocer las características específicas de los equipos que venden.

- Curso de vendedores. Enseña una serie de técnicas de ventas.

- Curso de literatura. Amplía el conocimiento literario al estudiar las sinopsis de los libros que venden (cada dos años).

- Curso de música. Se les brinda un panorama general de las disqueras y de los principales artistas.

- Imagen Cuba, arte en casa. Se pretende que los trabajadores asocien con sus creadores las pinturas que se ilustran en los productos, así como los aspectos que identifican a cada artista.

\section{Determinación de los conocimientos críticos}

Con la contribución de los gerentes de los departamentos Comercial, de Recursos Humanos, Centros Culturales y Economía se pudo efectuar un análisis exhaustivo de los conocimientos existentes por cada área de la empresa, de donde resultó el mapa de conocimientos de la sucursal ARTex de Villa Clara.

Los conocimientos críticos se determinaron a partir del nivel de influencia en los procesos clave, estos son:

- Habilidades para la venta. Los dependientes deben ser capaces de vender, de incitar a la compra con el uso de técnicas y habilidades que deben ir aprendiendo y adquiriendo en la práctica.

- Curso de dependientes integrales. Está compuesto por cinco cursos (técnico de reciclaje; de vendedores; literatura; música; e imagen Cuba, arte en casa), los cuales tienen como fin convertir a los vendedores en promotores culturales, en dependientes que puedan ser calificados como integrales; en primer lugar tienen que conocer y cumplir ciertas normas de comportamiento.

- Pericia de los expertos. Estos deben convertirse en una fuente de consulta para los trabajadores en la entidad, la sucursal cuenta con seis expertos.

- Manual de política comercial. Incluye todas las regulaciones, procedimientos y normas que directivos y trabajadores de las entidades pertenecientes a ARTex deben tener en cuenta. 
- Confección de nómina. El proceso de confección de nómina es el más representativo de la entidad, involucra a dos áreas muy importantes: recursos humanos y economía; influye además en la motivación del personal.

- Relaciones con artesanos. Estos suministran a la entidad artículos que son evaluados por un comité para certificar su calidad y comprobar que mantienen las características que debe poseer la mercancía ofertada por ARTex.

- Relaciones con el Ministerio de Turismo (Mintur). Esta institución se encarga de ofrecer capacitación a los trabajadores de los centros culturales, adiestrándolos en gastronomía e idiomas.

- Relaciones con los clientes. El comprador de un producto en un establecimiento espera cierto número de prestaciones antes, durante y después de la compra: limpieza, orden, amabilidad, información, garantía; de ahí que la calidad en la atención al cliente dependa directamente de los trabajadores y de las gestiones que hagan para complacer a sus clientes.

\section{Evaluación del conocimiento}

A partir de los aspectos privativos de la empresa se aplicaron las diferentes técnicas de evaluación del conocimiento para determinar hasta qué grado su estado puede influir en el desempeño de la organización. La entidad está compuesta por cuatro dependientes, un economista y el administrador.

Para evaluar las habilidades se tomó como referencia el punto de venta de ARTex Mi Ilusión; se analizó un conjunto de habilidades que deben poseer los dependientes en aras de lograr una adecuada atención al cliente.

- Nivel de conocimiento de los productos que vende.

- Uso de técnicas de marketing.

- Agilidad y astucia.

- Capacidad para incitar a los clientes a la compra.

- Aspecto personal.

Las evaluaciones se realizaron considerando el criterio de su administrador, quien representa el cargo de mayor nivel en esta entidad. 
Cuadro 3. Habilidades de venta en el punto de venta Mi Ilusión

\begin{tabular}{|c|c|c|c|c|c|c|c|}
\hline \multirow{2}{*}{$\begin{array}{l}\text { NOMBRE Y } \\
\text { APELLIDOS }\end{array}$} & \multirow[t]{2}{*}{ OCUPACIÓN } & \multicolumn{5}{|c|}{ HABILIDADES } & \multirow{2}{*}{$\begin{array}{l}\text { MEDIA POR } \\
\text { TRABAJADOR }\end{array}$} \\
\hline & & 1 & 2 & 3 & 4 & 5 & \\
\hline Martha Cordero & Cajera, dependiente & 4 & 3 & 4 & 4 & 5 & 4 \\
\hline Mónica Órea & Dependiente & 4 & 3 & 4 & 4 & 5 & 4 \\
\hline Yadira Machado & Dependiente & 4 & 3 & 4 & 4 & 5 & 4 \\
\hline Danny Miliano & Cajera, dependiente & 4 & 3 & 4 & 4 & 5 & 4 \\
\hline William Nazco & Administrador & 5 & 5 & 5 & 5 & 5 & 5 \\
\hline $\begin{array}{l}\text { Media por } \\
\text { habilidad }\end{array}$ & & 4.2 & 3.4 & 4.2 & 4.2 & 5 & \\
\hline
\end{tabular}

Fuente: Elaboración propia.

La aplicación del instrumento puso de manifiesto que el promedio de las habilidades de venta es muy bueno, aunque su tendencia es ir incrementando a medida que se realizan los cursos planificados por la sucursal ARTex de Villa Clara. Se necesita prestar atención al uso de las técnicas de mercadotecnia y, por su importancia, a la capacidad para incitar la compra en los clientes.

Para evaluar la formación en la empresa se aplicó un test en el que se escogió también el punto de venta Mi Ilusión por disponer del mayor número de personas que han pasado el curso de formación de dependientes integrales y por ser uno de los sitios con mayores índices de ventas. El curso de dependientes integrales está compuesto a su vez por cinco cursos:

1. Curso técnico de reciclaje.

2. Curso de vendedores.

3. Curso de literatura.

4. Curso de música.

5. Imagen Cuba, arte en casa. 
Cuadro 4. Impacto de los cursos

\begin{tabular}{l|l|l|l|l|l|c|c|c|}
\hline Nombre DEL TRABAJADOR & Función & \multicolumn{9}{|c|}{ CURSOS } \\
\cline { 2 - 9 } & & 1 & 2 & 3 & 4 & 5 & $\begin{array}{c}\text { DEPENDIENTES } \\
\text { INTEGRALES }\end{array}$ \\
\hline Mónica Órea & Trabajador & 4 & 5 & 3 & 4 & 5 & 5 \\
Martha Cordero & Trabajador & 4 & 5 & 3 & 4 & 5 & 5 \\
Yadira Machado & Trabajador & 5 & 5 & 3 & 4 & 5 & 5 \\
Danny Miliano & Trabajador & 4 & 5 & 3 & 5 & 5 & 5 \\
William Nazco & Evaluador & 5 & 5 & 4 & 4 & 5 & 5 \\
\hline
\end{tabular}

Fuente: Elaboración propia.

El curso de dependientes integrales posee un gran impacto dentro del proceso de compra y venta. Los trabajadores consideran que, en su caso, el de literatura es el de menor impacto debido a que no ofertan libros, pero admiten su valor como elemento de su formación integral. Se evidencia que cada trabajador, a pesar de rotar por todas las áreas de la tienda, se identifica con una en específico y, por tanto, le atribuyen un mayor nivel de impacto.

Para la evaluación de la pericia se tuvo en cuenta a los expertos formales e informales, en total seis con amplio dominio del tema a que se dedican.

\section{Cuadro 5. Evaluación de la experticia}

\begin{tabular}{|c|c|c|c|c|c|c|c|}
\hline \multirow[t]{2}{*}{ CRITERIOS } & \multicolumn{6}{|c|}{ EXPERTOS } & \multirow[t]{2}{*}{ MEDIA POR CRITERIO } \\
\hline & 1 & 2 & 3 & 4 & 5 & 6 & \\
\hline 1 & 5 & 5 & 5 & 5 & 4 & 4 & 4.7 \\
\hline 2 & 5 & 4 & 5 & 5 & 2 & 5 & 4.3 \\
\hline 3 & 5 & 2 & 5 & 4 & 5 & 4 & 4.2 \\
\hline 4 & 5 & 2 & 4 & 5 & 4 & 3 & 3.8 \\
\hline 5 & 5 & 5 & 5 & 5 & 2 & 5 & 4.5 \\
\hline Media por experto & 5 & 3.6 & 4.8 & 4.8 & 3.4 & 4.2 & \\
\hline
\end{tabular}

Fuente: Elaboración propia. 
A su vez, se determinó evaluar un manual y para tal fin se escogió el de política comercial, que sirve de guía a los gerentes al momento de informar a sus subordinados y a todos los que trabajan en el área comercial.

Cuadro 6. Análisis del manual de política comercial

\begin{tabular}{|c|c|c|c|c|}
\hline \multirow[t]{2}{*}{ USUARIOS DEL MANUAL } & \multicolumn{4}{|c|}{ MEDIA DE APROVECHAMIENTO DEL MANUAL } \\
\hline & Contenido & Dominio & $\begin{array}{l}\text { ACCESIBILIDAD Y } \\
\text { UTILIZACIÓN }\end{array}$ & $\begin{array}{c}\text { TOTAL POR } \\
\text { USUARIO }\end{array}$ \\
\hline Cándido Rivas Pérez & 5 & 4 & 5 & 4.6 \\
\hline Yudania Márquez Cabrera & 5 & 3.5 & 5 & 4.5 \\
\hline Juan Fernández Fernández & 5 & 4 & 5 & 4.6 \\
\hline Eric Estrada Martínez & 5 & 3 & 5 & 4.3 \\
\hline Tania Hernández Camuza & 5 & 3.6 & 4.5 & 4.3 \\
\hline Yuliet Mujica Jiménez & 5 & 4 & 5 & 4.6 \\
\hline Felipe Hernández Moya & 5 & 3.5 & 4.5 & 4.3 \\
\hline Regina Herrera Hernández & 5 & 4 & 5 & 4.6 \\
\hline Abel Lorenzo Calzadilla & 5 & 4.3 & 5 & 4.7 \\
\hline Jesús Monteagudo Seijo & 5 & 4 & 5 & 4.6 \\
\hline Media por dimensión & 5 & 3.79 & 4.9 & \\
\hline
\end{tabular}

Fuente: Elaboración propia.

Entre las recomendaciones referentes al manual debe destacarse el hecho de que no tiene un índice que oriente a quien desee consultarlo. El manual es considerado muy extenso, se aprecian muchas regulaciones ajenas al personal de la entidad y se propone separar este por departamentos. En las encuestas se evidencia su excelente nivel de accesibilidad y un contenido claro y específico. El mayor problema está en su dominio debido a que no se han realizado capacitaciones que adiestren a los afectados en el uso de este manual de política comercial. 
Se determinó evaluar el proceso de confección de nómina, el cual comienza en el área de recursos humanos, que es la encargada de elaborar el modelo SC4-05 prenómina con base en la información brindada por todas las áreas de la empresa y relacionada con vacaciones, licencias, ausencias u otra información del trabajador; después se entrega al área de contabilidad, que efectúa las retenciones que correspondan y emite el cheque para el pago de la nómina (esto, una vez que la persona autorizada en el modelo 09-03-53 "Personas autorizadas a solicitar cheques” se lo pide). Por último, el área de contabilidad se encarga de comprobar que el cuadre de las hojas y el total coincidan con el importe del cheque emitido.

Se estableció que las dificultades esenciales están en la entrega a destiempo de la información necesaria y en la disponibilidad de uno de los programas del sistema integral Zeus (confección de nómina), el cual minimiza el tiempo de este proceso. Los principales responsables de este proceso poseen una vasta experiencia enriquecida por los años y por las capacitaciones recibidas. Los procesos de comunicación, control y dirección se llevan a cabo de manera excelente y se reflejan en la efectividad del proceso en análisis.

También se evaluaron las relaciones externas con determinados actores. ARTex les brinda a sus trabajadores, fundamentalmente a los de centros culturales, una capacitación impartida por el Mintur, la cual constituye uno de los logros más significativos de la entidad. En estas se les enseña a ser unos gastrónomos eficientes, se les brinda un curso intensivo de idioma y profundizan en aspectos asociados con la cultura y las costumbres de los extranjeros. Todos estos cursos se coordinan por medio de recursos humanos y se realizan en el Mintur o en alguna otra institución perteneciente a la cadena de tiendas de ARTex. Para llevar a cabo este intercambio se cuenta con la colaboración de la relacionista, los gerentes general y comercial de la sucursal de Villa Clara, y además con una representante de Paradiso. Se tuvieron en cuenta siete criterios.

Según los resultados del cuestionario, los más involucrados en la relación con el Mintur tienen un nivel de conocimiento muy bueno de todos los requerimientos implícitos en esta relación. Algunos criterios, como el aprovechamiento de los elementos que favorecen la relación y sus posibilidades, deben llevarse a un nivel superior debido a su importancia y al beneficio que traerían si esas posibilidades se explotaran al cien por ciento. 
Cuadro 7. Relaciones de ARTex con el Mintur

\begin{tabular}{|c|c|c|c|c|c|c|c|c|}
\hline \multirow[t]{2}{*}{ NOMBRE DEL TRABAJADOR } & \multirow[t]{2}{*}{ CARGOS } & \multicolumn{7}{|c|}{ CRITERIOS } \\
\hline & & 1 & 2 & 3 & 4 & 5 & 6 & 7 \\
\hline Lisandra Puentes & Relacionista & 5 & 4 & 4 & 4 & 4 & 4 & 4 \\
\hline Jorge L. Santana Porto & Gerente General & 5 & 5 & 5 & 4 & 4 & 5 & 5 \\
\hline Juan Fernández Fernández & Gerente Comercial & 5 & 5 & 5 & 4 & 5 & 5 & 5 \\
\hline Nancy Lugo & Gerente de Paradiso & 5 & 5 & 4 & 4 & 4 & 4 & 4 \\
\hline Abel Lorenzo Calzadilla & $\begin{array}{l}\text { Gerente de Recursos } \\
\text { Humanos }\end{array}$ & 5 & 5 & 5 & 4 & 4 & 5 & 4 \\
\hline Media por criterio & & 5 & 4.8 & 4.6 & 4 & 4.2 & 4.6 & 4.4 \\
\hline
\end{tabular}

Fuente: Elaboración propia.

Para medir la lealtad de los clientes se utilizó la encuesta como instrumento. Para definir un cliente leal se consideraron siete criterios. La cantidad de clientes a encuestar fue calculada de acuerdo con el modelo binomial y se aplicó sobre la base de que los encuestados son clasificados como clientes especializados. Los criterios de lealtad de los clientes son:

1. Prefiere la atención del personal de venta de la empresa.

2. La atención que recibe le incita a volver a demandar el servicio.

3. Los bienes que ofertan son su primera opción.

4. Comunica a otros clientes su satisfacción con la oferta de la empresa.

5. Las diferencias de precios con otras empresas no limita su compra por la calidad que representa ARTex.

6. Confía en la gestión eficiente y oportuna de la empresa para cubrir su demanda.

7. Acude a los puntos de venta de la empresa para su compra. 
Los resultados evidencian la desconfianza de los clientes en cuanto a la gestión eficiente y oportuna de los productos, lo que en gran medida no depende directamente de la sucursal sino de los proveedores extranjeros y de los trámites burocráticos indispensables para que los productos entren al país. Además, se puede apreciar que el público es muy sensible al precio, es decir, la calidad que representan los productos de ARTex, que no son de primera necesidad, no opaca que la mayoría de las veces el cliente se incline hacia productos sustitutos o simplemente compre estos a la competencia debido a la posible diferencia de precios.

ANÁLISIS DEL ESTADO DEL CONOCIMIENTO EN LA EMPRESA

A partir de los análisis realizados se determinaron las fortalezas y debilidades de la empresa en términos de conocimiento.

Fortalezas:

- Los recursos humanos presentan amplia experiencia, profesionalidad y sentido de pertenencia.

- Oferta de productos exclusivos tanto para la población como para el turismo.

- Los expertos son reconocidos y consultados por toda la empresa y sus conocimientos sirven de apoyo al elaborar cualquier estrategia con miras al crecimiento organizacional.

- Los directivos de la sucursal ARTex de Villa Clara califican las habilidades de venta de sus trabajadores como muy buenas.

- Los procesos de la entidad se desarrollan eficientemente debido al nivel de especialización que han adquirido.

- La entidad posee un cronograma de capacitación flexible y adaptable a los cambios.

- Las relaciones con sus proveedores y agentes externos son muy sólidas, posibilitando que los procesos claves de la organización no sean afectados por posibles desavenencias.

- Aprovechamiento, en gran medida, de la relación entre ARTex y el Mintur.

Debilidades:

- Falta de abastecimiento de los productos que dependen del proceso de importación, debido a que ARTex posee proveedores extranjeros como China. 
- No utilizan a plenitud el manual guía de política comercial, que brinda una serie de procedimientos que podrían optimizar el tiempo y el esfuerzo de los trabajadores.

- Poco uso de las técnicas de mercadotecnia por los trabajadores, lo que dificulta el proceso de compra y venta.

- El cliente no se siente satisfecho del todo con el personal en contacto de la entidad.

- No existe confianza en la gestión eficiente y oportuna de ARTex.

\section{Estrategia de gestión del conocimiento}

A partir de los resultados de la etapa anterior se desarrolló una estrategia para atenuar las debilidades y potenciar las fortalezas de la empresa.

OBJetivos

- Aprovechar la especialización y la experiencia de algunos trabajadores, así como de los expertos para capacitar a los empleados.

- Capacitar en el uso de las técnicas de marketing con la finalidad de elevar el nivel de eficiencia en el proceso de compra y venta e incrementar a excelente el nivel de las habilidades de venta del personal en contacto de los puntos de venta.

- Incrementar la utilización y el conocimiento del manual de política comercial.

- Optimizar la relación con el Mintur para mejorar la atención al cliente en centros culturales y puntos de venta.

PLAN DE ACCIÓN

- Con el fin de incrementar la utilización del manual se podría crear un índice de contenido y reunir en documentos separados los aspectos más importantes a utilizar por departamentos, así se optimizaría el tiempo dedicado a buscar cualquier información específica en el manual.

- Dar a conocer las técnicas de marketing más comunes y viables a los trabajadores de los puntos de venta para que al menos tengan una noción de las posibles vías para incrementar la eficiencia en sus puestos de trabajo; después, estas podrían ser profundizadas y explicadas por algún experto 
en la materia mediante una capacitación de corta duración debido a que la esencia ya estaría dada.

- Ahondar en las posibilidades que ofrece la relación con el Mintur y, así, explotar al máximo esa unión.

- Analizar con los trabajadores de los puntos de venta los resultados del test de satisfacción del cliente, para que realicen cambios en aras de incrementar el grado de satisfacción con el personal en contacto y con la entidad en sentido general; esta sería una alternativa para aumentar el nivel de confianza de los clientes.

- Dar más publicidad a las promociones de los productos de ARTex y en las cuales no participan los más interesados: los clientes.

COMUNIDAD DE PRÁCTICA

Se propone desarrollar dos tipos de comunidades de práctica, una en la sucursal ARTex de Villa Clara y otra en los puntos de venta pertenecientes a esta sucursal. Sería muy ventajoso que los viernes se enviaran los temas a tratar por día en los puntos de venta, así se lograría uniformidad y organización, y estos podrían ser enviados por el responsable de capacitación de la sucursal ARTex de Villa Clara.

Comunidad de práctica para la sucursal ARTex de Villa Clara

- Participantes: todos los trabajadores de los departamentos de recursos humanos, economía, comercial y centros culturales.

- Temas a tratar: estado de las relaciones de la entidad con sus agentes externos; análisis de las normas, procedimientos y manuales que rigen el desarrollo de los procesos de la entidad; niveles de satisfacción del cliente y de los trabajadores; examen de las causas del crecimiento o disminución de las ventas.

- Horario y lugar: primero en las reuniones de los gerentes de las áreas y jefes de departamento con el gerente, y después en las reuniones de los departamentos. También se pueden tratar algunos temas en los encuentros matutinos y en las reuniones generales. 
Comunidad de práctica para los puntos de venta

- Participantes: todos los trabajadores del punto de venta.

- Temas a tratar: deberán ser enviados por la sucursal con un cronograma para que todos los puntos aborden simultáneamente el mismo tema, que debe versar sobre la base de los siguientes aspectos: nivel de información de los productos que se ofrecen; relación con el cliente; grado de agilidad y destreza en la atención al cliente y en la manipulación de la mercancía; organización del área de trabajo y diseño del punto de venta; aspecto personal y comportamiento en el trabajo.

- Horario y lugar: se pueden tomar algunos minutos del encuentro matutino convocado diariamente por el gerente del punto de venta.

DIRECTORIO DE EXPERTOS

La experiencia y la pericia deben ser explotadas por todos los miembros de una organización; el directorio es una herramienta de localización de los expertos de la entidad; este debe estar accesible para la consulta y el aprendizaje. Se creó un directorio de expertos de la empresa y se puso al alcance de todos los trabajadores con necesidades de conocimiento.

APROBACIÓN Y APLICACIÓN DE LA ESTRATEGIA

Se consultó con el responsable de capacitación y cuadros, quien confirmó la viabilidad de la estrategia y de la utilización de las técnicas propuestas, explotando el directorio de expertos y las comunidades de práctica. Se aplicará la estrategia con la premisa de que es una de las vías para incrementar el conocimiento de los trabajadores y, en consecuencia, la eficiencia de la cadena de tiendas de ARTex. Para su desarrollo es imprescindible el apoyo, ya confirmado, de todos los jefes de departamento, ya que son los responsables de controlar las actividades asignadas a sus trabajadores.

Los métodos empleados fueron sobre todo la observación y las encuestas. A los trabajadores del punto de venta Mi Ilusión se les recordó su reglamento y se les ofrecieron opciones para lograr una venta efectiva. Fue muy útil enseñarles los resultados de los test aplicados porque se percataron de los criterios brindados por los clientes. Se les mostró su importancia en el proceso de venta y cómo su conducta puede beneficiar o perjudicar directamente los resultados de la entidad. Siempre se destacaron sus capacidades y habilidades. 
Por otra parte, con el responsable de capacitación y cuadros de la entidad se analizaron los resultados obtenidos y la estrategia a seguir, comenzando por la publicación del directorio de expertos y las sugerencias de cursos de capacitación que se deben planificar.

\section{Evaluación del conocimiento organizacional}

Los test de satisfacción del cliente se aplicaron a una muestra de clientes especializados. De acuerdo con los datos del test, es válido afirmar que el grado de satisfacción es bueno. Corroborando lo expuesto, el cliente expone que el nivel de información que se le ofrece acerca de los productos es regular, lo cual, como es lógico, obstaculiza el proceso de venta, pues es una de las armas fundamentales de las cuales debe valerse el vendedor. Sin embargo, hay que tener en cuenta que las demás dimensiones reportaron resultados buenos y con tendencia a muy buenos; de hecho, considerando los criterios emitidos por los clientes, no solo es posible obtener una evaluación de muy bien sino incluso alcanzar la excelencia.

Coeficiente de satisfacción con la atención al cliente (CSac)

$$
\begin{aligned}
& \text { CSac }=0.3 M \\
& \text { CSac }=1.0656
\end{aligned}
$$

Atributos a tener en cuenta para aplicar el test de satisfacción con la gestión de la oferta:

- Calidad

- Precio

- Disponibilidad

- Accesibilidad

- Funcionalidad

El test presentado comprobó resultados ya sospechados: a pesar de que los clientes consideran que los artículos de ARTex son de buena calidad encuentran 
que los precios son regulares; se puede apreciar la aparición de nuevas variables que influyen mucho en el criterio de compra: una, ajena a la entidad, es la accesibilidad, y la otra depende por completo del proceso de gestión de los productos (proceso que el cliente no ubica en buena posición) y es la disponibilidad. El hecho de que esta variable no se encuentre en óptimas condiciones es una de las principales causas por las cuales el cliente no es leal y simplemente busca productos sustitutos en la competencia, aspecto lamentable que debe ser erradicado.

Coeficiente de satisfacción con la gestión de la oferta (Csgo)

$$
\begin{aligned}
& C \text { Sgo }=0.5 M \\
& \text { CSgo }=1.595
\end{aligned}
$$

El punto de venta Mi Ilusión es considerado por los clientes como uno de los mejores si de ambiente general se trata. La insatisfacción con este fue encontrada en cuanto a las promociones y la disponibilidad. El diseño del punto de venta en general fue clasificado como bueno pero con tendencia al mejoramiento, lo que podría ocurrir muy rápido si se incorporan las propuestas de los clientes.

Coeficiente de satisfacción con el diseño del punto de venta (CSpv)

$$
\begin{aligned}
& C S p v=0.2 M \\
& C S p v=0.696
\end{aligned}
$$

Coeficiente de satisfacción general (CS)

$$
\begin{gathered}
C S=C S a c+C S g o+C S p v \\
C S=3.3566
\end{gathered}
$$


En general, el nivel de satisfacción del cliente es bueno, el proceso más complicado (y el más difícil de solucionar y medir) es el de gestión de la oferta, al que se le asigna la mitad del peso al hablar de satisfacción del cliente. Esto no quiere decir que se desatiendan los demás procesos, por el contrario, se hace imprescindible incrementar al máximo el nivel de satisfacción con el diseño del punto de venta y con la atención al cliente, ya que las vías para solucionar los problemas encontrados en estos son más accesibles y con resultados más rápidos y visibles, pero no en el caso de la gestión de la oferta, que exige soluciones más extensas.

Es indispensable aclarar que la estrategia fue aplicada en un solo punto de venta tomado como piloto de la investigación, y el periodo analizado, después de puesta en marcha la estrategia, fue prudencial. Pero ese tiempo bastó para ratificar todo lo expuesto sobre la necesidad de la gestión del conocimiento en las empresas, en este caso las pertenecientes a ARTex, pues se notó un cambio en los criterios de los clientes especializados acerca de la atención al cliente y del diseño del punto de venta, el incremento en la media en el test de satisfacción del cliente fue de 3.8 \% aproximadamente, y en el test de diseño del punto de venta se pudo constatar un aumento de alrededor de $3.1 \%$, estos cambios son mucho más fáciles de percibir ya que se relacionan con el aspecto organizativo y estructural de la tienda, en la cual se puede realizar todo tipo de cambio siempre y cuando sea para mejorar la opinión de los clientes.

El test de gestión de la oferta no fue aplicado porque en el periodo de prueba no hubo entradas sustanciales de mercancías 0 , al menos, cambios de los cuales los clientes pudieran percatarse. Es importante aclarar que, no obstante que el test de gestión de la oferta no fue aplicado con los cambios sustanciales en satisfacción del cliente y diseño del punto de venta, el coeficiente general fue de 3.42, $1.9 \%$ superior al valorado antes de implementar la estrategia.

A partir de los documentos facilitados por la sucursal ARTex de Villa Clara y por el punto de venta Mi Ilusión, se efectuó un análisis costo-beneficio en el cual se expusieron las ventajas de aplicar el procedimiento para la gestión del conocimiento, con resultados muy favorables materializados con un incremento de $5 \%$ en las ventas. Este incremento, que se comenzará a apreciar a partir del mes en que inició la estrategia, traerá consigo un aumento considerable de 112 lo planificado para el periodo. Lo expuesto hasta ahora se puede observar en los cuadros 8 y 9 . 
Cuadro 8. Distribución de los costos

INVERSIONES DE LA PUESTA EN MARCHA DEL PROCEDIMIENTO

Provenientes de la sucursal ARTex de Villa Clara:

Impreso y papeles

Accesorios de computación

Consumo de energía eléctrica

Teléfono y fax

Servicio de internet y correo

Diseños (promoción)

Materiales para promoción (nivel nacional)

Capacitación

Transporte

Gastos totales planificados en el punto de venta

Total de gastos
IMPORTE (\$ CUC)

1626

751

175

100

85

105.50

40

50

250

70

14605.63

16231.63

Fuente: Elaboración propia con base en documentos de la entidad.

\section{Retroalimentación de las etapas anteriores}

La gestión del conocimiento debe ser dinámica y reconstructiva, así que los problemas que pudieran encontrarse durante la puesta en marcha de la estrategia deben servir como base para el mejoramiento continuo y la implantación de nuevos objetivos con sus respectivas tareas y, por tanto, la reorientación de la estrategia.

\section{Consideraciones finales}

La gestión del conocimiento tiene una gran relevancia en el contexto de las empresas de comercio minorista de bienes en Cuba por su alto contenido de intangibilidad. El procedimiento que se propone para la sucursal ARTex de Villa Clara es una guía de pasos que busca implementar la gestión del conocimiento en una empresa de su tipo para potenciar la línea de negocio del comercio minorista de bienes. 
Cuadro 9. Beneficios del punto de venta Mi Ilusión

\begin{tabular}{|c|c|}
\hline PARTIDAS & IMPORTE (\$ CUC) \\
\hline Ventas históricas promedio (2008-abril de 2012) & 297188.45 \\
\hline Hasta diciembre de 2008 & 346381 \\
\hline Hasta diciembre de 2009 & 350043 \\
\hline Hasta diciembre de 2010 & 254286 \\
\hline Hasta diciembre de 2011 & 272210 \\
\hline Hasta abril de 2012 & 63906 \\
\hline Total de ventas esperadas en 2012 & 274004 \\
\hline Incremento de las ventas por la aplicación de la estrategia $(5 \%)^{1}$ & 13700.02 \\
\hline Total de ventas esperadas luego de la aplicación de la estrategia & 223798.02 \\
\hline $\begin{array}{l}\text { Total de ventas esperadas para } 2012 \text { (bajo la influencia de la } \\
\text { estrategia) }\end{array}$ & 287704.02 \\
\hline $\begin{array}{l}\text { Ingresos esperados del punto de venta luego de la aplicación de la } \\
\text { estrategia }\end{array}$ & 273098.57 \\
\hline Utilidad de la investigación & 271472.57 \\
\hline
\end{tabular}

${ }^{1}$ Dato suministrado por la experta en economía de la entidad, a partir de documentos de la empresa

Fuente: Elaboración propia con base en los documentos del punto de venta.

Para continuar este trabajo debe darse seguimiento a los resultados de la puesta en práctica de la estrategia considerando que debe existir un proceso de retroalimentación producto de los cambios posibles en el entorno. Además, debe profundizarse en las causas de los aspectos de la gestión de la oferta que dejan insatisfechos a los clientes, para cubrir plenamente sus necesidades y expectativas y generalizar el procedimiento en las restantes unidades comerciales pertenecientes al comercio minorista de bienes, adaptándolo a los requerimientos y exigencias de cada una. 


\section{Fuentes consultadas}

Alzate Molina, C.A. y G.A. Gallego Álvarez (2012). "Gestión del conocimiento" [en línea]. Medellín: Biblioteca Digital Universidad de San Buenaventura. Disponible en: http://hdl.handle.net/10819/1058

Benavides Velasco, C.A. y C. Quintana García (2003). Gestión del conocimiento y calidad total. Madrid: Ediciones Díaz de Santos.

García Morales, V.J. (2005). "Análisis de las barreras del aprendizaje organizacional desde la perspectiva de los centros educativos". Dirección y Organización, 31, febrero, 50-67.

La Fé Jiménez, R.Y. (2014). "La gestión del conocimiento en empresas de comercio minorista de bienes en Villa Clara, Cuba”. Teoría y Praxis [en línea], (15), 9-35. Disponible en: http://www.teoriaypraxis.uqroo.mx/ doctos/numero15/La-Fe.pdf

Núñez Paula, I. y Y. Núñez Govín (2005). "Propuesta de clasificación de las herramientas - software para la gestión del conocimiento”. Acimed [en línea], (13) 2. Disponible en: http://bvs.sld.cu/revistas/aci/vol13_2_05/ aci03_05.htm

Ollarves Levison, Y., M. Estévez González y L.A. Salguero (2012). "Propuesta para construcción de un modelo de gestión del conocimiento en una unidad de planificación y desarrollo de la UPEL”. Una Investig@ción, IV (8), 13-41.

Orozco Silva, E. y J.R. Carro Suárez (2002). "Propuesta de estrategia para la introducción de la gestión de la información y la gestión del conocimiento en las organizaciones cubanas”. Ciencias de la Información, 33 (1), abril, 17-23.

Pérez Fernández, E. (2002). "Gestión del conocimiento en organizaciones geográficamente dispersas”. Dirección y Organización, 27, febrero, 14-24.

Ponjuán Dante, G. (2006). Introducción a la gestión del conocimiento. La Habana: Félix Valera.

Rezende, Y. (2001). "Información para los negocios: los nuevos agentes del conocimiento y la gestión del capital intelectual”. Ciencias de la Información, 32 (2), mayo-agosto, 47-57.

Riesco González, M. (2006). El negocio es el conocimiento. Madrid: Ediciones Díaz de Santos. 
